\title{
Some Results on Hermite-Hadamard Type Inequality through Convexity
}

\author{
Shahid Qaisar, Sabir Hussain* \\ ${ }^{1}$ College of Mathematics and Statistics, Chongqing University, Chongqing, P. R. China \\ ${ }^{2}$ Department of Mathematics, College of Science, Qassim University, Buraydah, Saudi Arabia \\ *Corresponding author: sabiriub@yahoo.com
}

Received March 05, 2014; Revised April 13, 2014; Accepted April 21, 2014

\begin{abstract}
Our aim in this article to establish various inequalities for some differentiable mapping that are connected with illustrious Hermite-Hadamard integral inequality for mapping whose absolute values of derivatives are convex. The new integral inequalities are then applied to some special means and as well as numerical integration to obtain some better estimates.
\end{abstract}

Keywords: Hermite-Hadamard type inequality, convex functions, special means, Hölder's integral inequality, power-mean inequality, Quadrature formula

Cite This Article: Shahid Qaisar, and Sabir Hussain, "Some Results on Hermite-Hadamard Type Inequality through Convexity." Turkish Journal of Analysis and Number Theory, vol. 2, no. 2 (2014): 53-59. doi: 10.12691/tjant-2-2-5.

\section{Introduction}

The role of mathematical inequalities within the mathematical branches as well as in its various application should not be underestimated. The appearance of the new mathematical inequality often puts on the firm foundation for the heuristic algorithms and techniques utilized within applied sciences. Among other one of the main inequality, which provides for us an explicit error bounds in the trapezodial and midpoint rules of a smooth function, called Hermite-Hadamard's inequality defined as [[1], p. 53]:

$$
f\left(\frac{a+b}{2}\right) \leq \frac{1}{b-a} \int_{a}^{b} f(x) d x \leq \frac{f(a)+f(b)}{2}
$$

where $f:[a, b] \rightarrow R$ is a convex function. Both inequalities hold in the reversed direction for $f$ to be concave. We note that Hermite-Hadamard's inequality may be regarded as a refinement of the concept of convexity and it follows easily from Jensen inequality. Inequality (1.1) has received renewed attention in recent years and a remarkable variety of refinements and generalization have been discovered $[2,7,10,11]$ and the refrences cited therein.

Theorem 1.1. ([3]) Let $f: I \subset R \rightarrow R$ be a differentiable function on $I^{0} \quad$ (interior of $I^{0}$ ) $a, b \in I$ with $a<b$. if the mapping $\left|f^{\prime}\right|$ is convex on $[a, b]$, and then we have the following inequality:

$$
\begin{aligned}
& \left|\frac{f(a)+f(b)}{2}-\frac{1}{b-a} \int_{a}^{b} f(x) d x\right| \\
& \leq \frac{(b-a)\left(\left|f^{\prime}(a)\right|+\left|f^{\prime}(b)\right|\right)}{8} .
\end{aligned}
$$

Theorem 1.2. ([3]) Let $f: I \subset R \rightarrow R \quad$ be a differentiable function on $I^{0} \quad$ (interior of $I^{0}$ ) $\quad a, b \in I$ with $a<b$. if the mapping $\left|f^{\prime}\right|^{p}$ is convex on $[a, b]$, for some fixed $p>1$ and then we have the following inequality:

$$
\begin{aligned}
& \left|\frac{f(a)+f(b)}{2}-\frac{1}{b-a} \int_{a}^{b} f(x) d x\right| \\
& \leq \frac{b-a}{2(p+1) \frac{1}{p}}\left[\frac{\left|f^{\prime}(a)\right| \frac{p}{p-1}+\left|f^{\prime}(b)\right| \frac{p}{p-1}}{2}\right] .
\end{aligned}
$$

In similar manner to Dragomir and Agarwal methodology, inequalities for differentiable convex mappings associated with the left-hand side of HermiteHadamard's (midpoint) inequality was verified by Kirmaci ,by means of the following illustration:

Theorem 1.3. ([5]) Let $f: I \subset R \rightarrow R \quad$ be a differentiable mapping on $I^{*}, a, b \in I^{*}$ with $a<b$. If $\left|f^{\prime}\right|$ is convex on $[a, b]$ then we have:

$$
\begin{aligned}
& \left|\frac{1}{b-a} \int_{a}^{b} f(x) d x-f\left(\frac{a+b}{2}\right)\right| \\
& \leq \frac{(b-a)\left(\left|f^{\prime}(a)\right|+\left|f^{\prime}(b)\right|\right)}{8} .
\end{aligned}
$$

Theorem 1.4. ([4]) Let $f: I \subset R \rightarrow R \quad$ be a differentiable function on $I^{0} \quad$ (interior of $I^{0}$ ) $a, b \in I$ with $a<b$. If the mapping $\left|f^{\prime}\right|^{p}$ is convex on $[a, b]$, for some fixed $\mathrm{p}>1$, and then we have the following inequality: 


$$
\begin{aligned}
& \left|\frac{1}{b-a} \int_{a}^{b} f(x) d x-f\left(\frac{a+b}{2}\right)\right| \\
& \leq(b-a)\left(\frac{3^{1-1 / p}}{8}\right)\left[\left|f^{\prime}(a)\right|+\left|f^{\prime}(b)\right|\right] .
\end{aligned}
$$

Theorem 1.5. ([4]) Let $f: I \subset R \rightarrow R$ be a differentiable function on $I^{0}$ (interior of $I^{0}$ ) $a, b \in I$ with $a<b$. If the mapping $\left|f^{\prime}\right|^{p}$ is convex on $[a, b]$, for some fixed $p \geq 1$, and $\left|f^{\prime}\right|$ is a linear map, we have the following inequality:

$$
\left|\frac{1}{b-a} \int_{a}^{b} f(x) d x-f\left(\frac{a+b}{2}\right)\right| \leq\left(\frac{b-a}{8}\right)\left|f^{\prime}(a+b)\right| .
$$

In most recent years, For additional findings relating to the Hermite-Hadamard integral inequality for utilizing different kind of convexity, readers are directed to ([1217]). This work is organized in the following way. After this Introduction, in Section 2 main results are presented.In Section 3 application to special means are considered. Finally Section 4, error is estimated for the generalized quadrature formula.

\section{Main results}

To prove our main result, we need some important lemma.

Lemma 2.1. Let $f:[a, b] \subset R \rightarrow R$ be differentiable function on $I^{0}$, where $a, b \in I$ is with $a<b$. If $f^{\prime} \in L([a, b])$. Then the following inequality holds:

$$
\begin{aligned}
& \frac{f\left(\frac{2 a+b}{3}\right)+f\left(\frac{a+2 b}{3}\right)}{2}-\frac{1}{b-a} \int_{a}^{b} f(x) d x \\
& =\frac{b-a}{9} \\
& {\left[\begin{array}{l}
\int_{0}^{1} \psi f^{\prime}\left(\psi \frac{2 a+b}{3}+(1-\psi) a\right) d \psi \\
+\int_{0}^{1}(\psi-1) f^{\prime}\left(\psi \frac{a+b}{2}+(1-\psi) \frac{2 a+b}{3}\right) d \psi \\
+\int_{0}^{1} \psi f^{\prime}\left(\psi \frac{a+2 b}{3}+(1-\psi) \frac{a+b}{2}\right) d \psi \\
+\int_{0}^{1}(\psi-1) f^{\prime}\left(\psi b+(1-\psi) \frac{a+2 b}{3}\right) d \psi
\end{array}\right] .}
\end{aligned}
$$

Proof. Using integrating by parts, and by making use of the substitution

$$
x=\psi \frac{2 a+b}{3}+(1-\psi) a
$$

we have

$$
\begin{aligned}
& \frac{b-a}{9} \int_{0}^{1} \psi f^{\prime}\left(\psi \frac{2 a+b}{3}+(1-\psi) a\right) d \psi \\
& =\frac{b-a}{9}\left[\left.\frac{3 \psi f^{\prime}\left(\psi \frac{2 a+b}{3}+(1-\psi) a\right)}{b-a}\right|_{0} ^{1}\right. \\
& =\left[\frac{1}{3} f\left(\frac{2 a+b}{3}\right)-\frac{1}{b-a} \int_{a}^{1} f\left(\psi \frac{2 a+b}{3}+(1-\psi) a\right) d \psi\right. \\
& \left.-\frac{(2 a+b) / 3}{b-a} f(x) d x\right],
\end{aligned}
$$

analougusoly,

$$
\begin{aligned}
& \frac{b-a}{9} \int_{0}^{1}(\psi-1) f^{\prime}\left(\psi \frac{a+b}{2}+(1-\psi) \frac{2 a+b}{3}\right) d \psi \\
& =\left[\frac{1}{3} f\left(\frac{2 a+b}{3}\right)-\frac{1}{b-a} \int_{(2 a+b) / 3}^{(a+b) / 2} f(x) d x\right], \\
& \frac{b-a}{9} \int_{0}^{1} \psi f^{\prime}\left(\psi \frac{a+2 b}{3}+(1-\psi) \frac{a+b}{2}\right) d \psi \\
& =\left[\frac{1}{3} f\left(\frac{a+2 b}{3}\right)-\frac{1}{b-a} \int_{(x+b) / 2}^{(x+2 b) / 3} f(x) d x\right], \\
& \frac{b-a}{9} \int_{0}^{1}(\psi-1) f^{\prime}\left(\psi b+(1-\psi) \frac{a+2 b}{3}\right) d \psi \\
& =\left[\frac{1}{3} f\left(\frac{a+2 b}{3}\right)-\frac{1}{b-a} \int_{(x+2 b) / 3}^{b} f(x) d x\right] .
\end{aligned}
$$

This proves as required.

Theorem 2.2. Let $f:[a, b] \subset R \rightarrow R \quad$ be a differentiable function on $I^{0}$, where $a, b \in I$ is with $a<b$. such that $f^{\prime} \in L([a, b])$. If the mapping $\left|f^{\prime}\right|$ is convex on $[a, b]$, then we have the following inequality:

$$
\begin{aligned}
& \left|\frac{f\left(\frac{2 a+b}{3}\right)+f\left(\frac{a+2 b}{3}\right)}{2}-\frac{1}{b-a} \int_{a}^{b} f(x) d x\right| \\
& \leq \frac{b-a}{54} \\
& {\left[\begin{array}{l}
\left|f^{\prime}(a)\right|+3\left|f^{\prime}\left(\frac{2 a+b}{3}\right)\right| \\
+2\left|f^{\prime}\left(\frac{a+b}{2}\right)\right|+3\left|f^{\prime}\left(\frac{a+2 b}{3}\right)\right| \\
+\left|f^{\prime}(b)\right|
\end{array}\right] .}
\end{aligned}
$$

Proof. Using Lemma 2.1 and taking the modulus, we have 


$$
\begin{aligned}
& \left|\frac{f\left(\frac{2 a+b}{3}\right)+f\left(\frac{a+2 b}{3}\right)}{2}-\frac{1}{b-a} \int_{a}^{b} f(x) d x\right| \leq \frac{b-a}{9} \\
& {\left[\begin{array}{l}
\int_{0}^{1} \psi\left|f^{\prime}\left(\psi \frac{2 a+b}{3}+(1-\psi) a\right)\right| d \psi \\
+\int_{0}^{1}(1-\psi)\left|f^{\prime}\left(\psi \frac{a+b}{2}+(1-\psi) \frac{2 a+b}{3}\right)\right| d \psi \\
+\int_{0}^{1} \psi\left|f^{\prime}\left(\psi \frac{a+2 b}{3}+(1-\psi) \frac{a+b}{2}\right)\right| d \psi \\
+\int_{0}^{1}(1-\psi)\left|f^{\prime}\left(\psi b+(1-\psi) \frac{a+2 b}{3}\right)\right| d \psi
\end{array}\right],}
\end{aligned}
$$

using the convexity of $\left|f^{\prime}\right|$, we have

$$
\begin{gathered}
\int_{0}^{1} \psi\left|f^{\prime}\left(\psi \frac{2 a+b}{3}+(1-\psi) a\right)\right| d \psi \\
=\frac{1}{3}\left|f^{\prime}\left(\frac{2 a+b}{3}\right)\right|+\frac{1}{6}\left|f^{\prime}(a)\right| \\
\int_{0}^{1}(1-\psi)\left|f^{\prime}\left(\psi \frac{a+b}{2}+(1-\psi) \frac{2 a+b}{3}\right)\right| d \psi \\
=\frac{1}{6}\left|f^{\prime}\left(\frac{a+b}{2}\right)\right|+\frac{1}{3}\left|f^{\prime}\left(\frac{2 a+b}{3}\right)\right|, \\
\int_{0}^{1} \psi\left|f^{\prime}\left(\psi \frac{a+2 b}{3}+(1-\psi) \frac{a+b}{2}\right)\right| d \psi \\
=\frac{1}{3}\left|f^{\prime}\left(\frac{a+2 b}{3}\right)\right|+\frac{1}{6}\left|f^{\prime}\left(\frac{a+b}{2}\right)\right|, \\
\int_{0}^{1}(1-\psi)\left|f^{\prime}\left(\psi b+(1-\psi) \frac{a+2 b}{3}\right)\right| d \psi \\
=\frac{1}{6}\left|f^{\prime}(b)\right|+\frac{1}{3}\left|f^{\prime}\left(\frac{a+2 b}{3}\right)\right| \cdot
\end{gathered}
$$

Combing the above inequalities (2.3), (2.4), (2.5), and (2.6), we obtain (2.2). This completes the proof.

Corollary 2.3 Under the conditions of Theorem 2.2,

$$
\begin{aligned}
& \left|\frac{f\left(\frac{2 a+b}{3}\right)+f\left(\frac{a+2 b}{3}\right)}{2}-\frac{1}{b-a} \int_{a}^{b} f(x) d x\right| \\
& \leq\left(\frac{b-a}{18}\right)\left[\left|f^{\prime}(a)\right|+\left|f^{\prime}(b)\right|\right] .
\end{aligned}
$$

Using the convexity of $\left|f^{\prime}(x)\right| \leq M$, for all $x \in[a, b]$, we have

$$
\left|\frac{f\left(\frac{2 a+b}{3}\right)+f\left(\frac{a+2 b}{3}\right)}{2}-\frac{1}{b-a} \int_{a}^{b} f(x) d x\right| \leq\left(\frac{b-a}{9}\right) M .
$$

Proof . The assertion follows from Theorem 2.2 and utilizing the convexity of $\left|f^{\prime}\right|$.

Theorem 2.4 Let $f:[a, b] \subset R \rightarrow R$ be a differentiable function on $I^{0}$, where $a, b \in I$ is with $a<b$. such that $f^{\prime} \in L([a, b])$. If the mapping $\left|f^{\prime}\right|^{q}$ is convex on $[a, b]$, then we have the following inequality :

$$
\begin{aligned}
& \left|\frac{f\left(\frac{2 a+b}{3}\right)+f\left(\frac{a+2 b}{3}\right)}{2}-\frac{1}{b-a} \int_{a}^{b} f(x) d x\right| \\
& \leq \frac{b-a}{9}\left(\frac{1}{p+1}\right)^{\frac{1}{p}}\left(\frac{1}{2}\right)^{\frac{1}{q}} \\
& {\left[\begin{array}{l}
\left(\left|f^{\prime}(a)\right|^{q}+\left|f^{\prime}\left(\frac{2 a+b}{3}\right)\right|^{q}\right)^{\frac{1}{q}} \\
\times\left(\left|f^{\prime}\left(\frac{a+b}{2}\right)\right|^{q}+\left|f^{\prime}\left(\frac{2 a+b}{3}\right)\right|^{q}\right)^{\frac{1}{q}} \\
+\left(\left|f^{\prime}\left(\frac{a+2 b}{3}\right)\right|^{q}+\left|f^{\prime}\left(\frac{a+b}{2}\right)\right|^{q}\right)^{\frac{1}{q}} \\
+\left(\left|f^{\prime}\left(\frac{a+2 b}{3}\right)\right|^{q}+\left|f^{\prime}(b)\right|^{q}\right)^{\frac{1}{q}}
\end{array}\right] .}
\end{aligned}
$$

Proof. Using Lemma 2.1 and Hölder inequality we have

$$
\begin{aligned}
& \left|\frac{f\left(\frac{2 a+b}{3}\right)+f\left(\frac{a+2 b}{3}\right)}{2}-\frac{1}{b-a} \int_{a}^{b} f(x) d x\right| \leq \frac{b-a}{9} \\
& {\left[\left(\int_{0}^{1} \psi^{p} d \psi\right)^{\frac{1}{p}}\left(\int_{0}^{1}\left|f^{\prime}\left(\psi \frac{2 a+b}{3}+(1-\psi) a\right)\right|^{q} d \psi\right)^{\frac{1}{q}}\right.} \\
& +\left(\int_{0}^{1}(1-\psi)^{p} d \psi\right)^{\frac{1}{p}}\left(\int_{0}^{1}\left|f^{\prime}\left(\begin{array}{l}
\psi \frac{a+b}{2} \\
+(1-\psi) \frac{2 a+b}{3}
\end{array}\right)\right|^{q} d \psi\right)^{\frac{1}{q}} \\
& +\left(\int_{0}^{1} \psi^{p} d \psi\right)^{\frac{1}{p}}\left(\int_{0}^{1} \mid f^{\prime}\left(\left.\begin{array}{l}
\psi \frac{a+2 b}{3} \\
+(1-\psi) \frac{a+b}{2}
\end{array}\right|^{q} d \psi\right)^{\frac{1}{q}}\right. \\
& {\left[+\left(\int_{0}^{1}(1-\psi)^{p} d \psi\right)^{\frac{1}{p}}\left(\int_{0}^{1}\left|f^{\prime}\left(+(1-\psi) \frac{a+2 b}{3}\right)\right|^{q} d \psi\right)^{\frac{1}{q}}\right]}
\end{aligned}
$$


using the convexity of $\left|f^{\prime}\right|$, we have

$$
\begin{aligned}
& \left.\int_{0}^{1}\left|f^{\prime}\left(\psi \frac{2 a+b}{3}+(1-\psi) a\right)\right|\right|^{q} d \psi \\
& \leq \frac{1}{2}\left|f^{\prime}\left(\frac{2 a+b}{3}\right)\right|^{q}+\frac{1}{2}\left|f^{\prime}(a)\right|^{q} \\
& \int_{0}^{1}\left|f^{\prime}\left(\psi \frac{a+b}{2}+(1-\psi) \frac{2 a+b}{3}\right)\right|^{q} d \psi \\
& \leq \frac{1}{2}\left|f^{\prime}\left(\frac{a+b}{2}\right)\right|^{q}+\frac{1}{2}\left|f^{\prime}\left(\frac{2 a+b}{3}\right)\right|^{q}, \\
& \int_{0}^{1}\left|f^{\prime}\left(\psi \frac{a+2 b}{3}+(1-\psi) \frac{a+b}{2}\right)\right|^{q} d \psi \\
& \leq\left.\frac{1}{2}\left|f^{\prime}\left(\frac{a+2 b}{3}\right)\right|\right|^{q}+\frac{1}{2}\left|f^{\prime}\left(\frac{a+b}{2}\right)\right|^{q}, \\
& \quad \frac{1}{2}\left|f^{\prime}\left(\psi b+(1-\psi) \frac{a+2 b}{3}\right)\right|^{q} d \psi \\
& \quad \frac{1}{0}\left|f^{\prime}(b)\right|^{q}+\frac{1}{2}\left|f^{\prime}\left(\frac{a+2 b}{3}\right)\right|^{q} .
\end{aligned}
$$

Combing the above inequalities (2.8), (2.9), (2.10), and (2.11), we obtain (2.7). This completes the proof.

Theorem 2.5 Let $f:[a, b] \subset R \rightarrow R$ be a differentiable function on $I^{0}$, where $a, b \in I$ is with $a<b$. such that $f^{\prime} \in L([a, b])$. If the mapping $\left|f^{\prime}\right|^{q}$ is convex on $[a, b]$, for some fixed $q \geq 1$, then we have the following inequality :

$$
\begin{aligned}
& \left|\frac{f\left(\frac{2 a+b}{3}\right)+f\left(\frac{a+2 b}{3}\right)}{2}-\frac{1}{b-a} \int_{a}^{b} f(x) d x\right| \\
& \leq \frac{b-a}{9}\left(\frac{1}{2}\right)^{\frac{1}{p}\left(\frac{1}{3}\right)^{\frac{1}{q}}} \mid \\
& \times\left[\begin{array}{l}
\left(\left|f^{\prime}(a)\right|^{q}+\left.2\left|f^{\prime}\left(\frac{2 a+b}{3}\right)\right|\right|^{q}\right)^{\frac{1}{q}} \\
+\left(\left|f^{\prime}\left(\frac{a+b}{2}\right)\right|^{q}+2\left|f^{\prime}\left(\frac{2 a+b}{3}\right)\right|^{q}\right)^{\frac{1}{q}} \\
+\left(2\left|f^{\prime}\left(\frac{a+2 b}{3}\right)\right|^{q}+\left|f^{\prime}\left(\frac{a+b}{2}\right)\right|^{q}\right)^{\frac{1}{q}} \\
+\left(2\left|f^{\prime}\left(\frac{a+2 b}{3}\right)\right|^{q}+\left|f^{\prime}(b)\right|^{q}\right)^{\frac{1}{q}}
\end{array}\right]
\end{aligned}
$$

Proof. Using Lemma 2.1 and power mean inequality we have

$$
\begin{aligned}
& \left|\frac{f\left(\frac{2 a+b}{3}\right)+f\left(\frac{a+2 b}{3}\right)}{2}-\frac{1}{b-a} \int_{a}^{b} f(x) d x\right| \leq \frac{b-a}{9}
\end{aligned}
$$

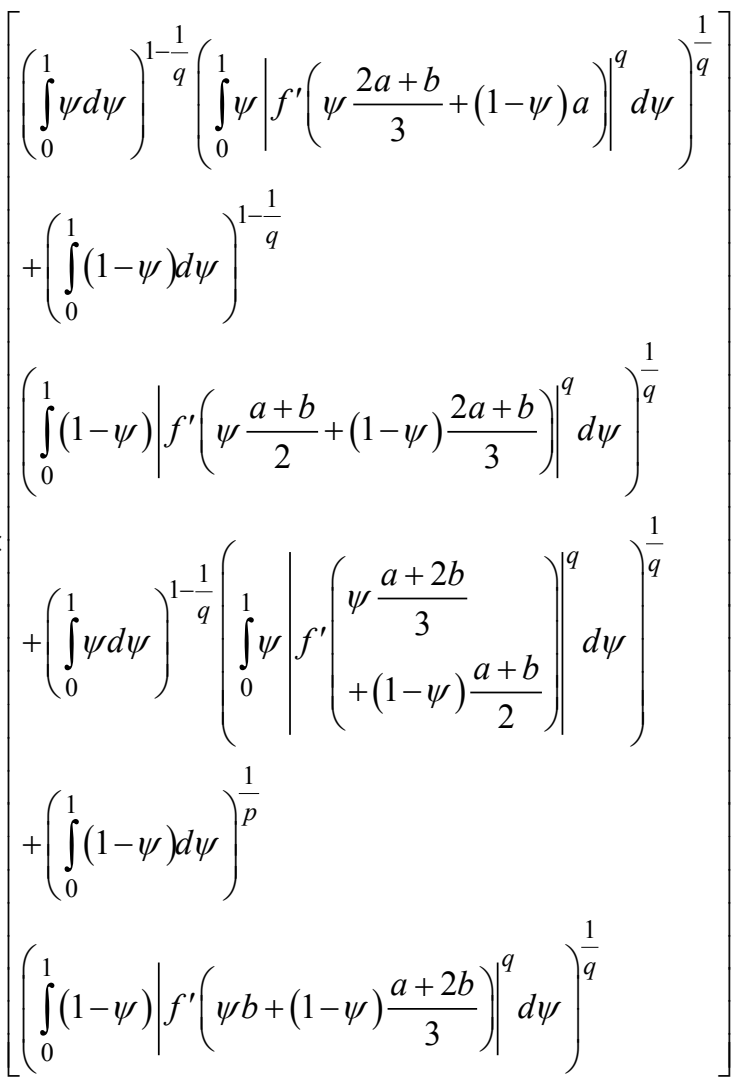

using the convexity of $\left|f^{\prime}\right|$, we have

$$
\begin{aligned}
& \int_{0}^{1} \psi\left|f^{\prime}\left(\psi \frac{2 a+b}{3}+(1-\psi) a\right)\right|^{q} d \psi \\
& \leq \frac{1}{3}\left|f^{\prime}\left(\frac{2 a+b}{3}\right)\right|^{q}+\frac{1}{6}\left|f^{\prime}(a)\right|^{q} \\
& \int_{0}^{1}(1-\psi)\left|f^{\prime}\left(\psi \frac{a+b}{2}+(1-\psi) \frac{2 a+b}{3}\right)\right|^{q} d \psi \\
& \leq\left.\frac{1}{6}\left|f^{\prime}\left(\frac{a+b}{2}\right)\right|\right|^{q}+\frac{1}{3}\left|f^{\prime}\left(\frac{2 a+b}{3}\right)\right|^{q}, \\
& \int_{0}^{1} \psi\left|f^{\prime}\left(\psi \frac{a+2 b}{3}+(1-\psi) \frac{a+b}{2}\right)\right|^{q} d \psi \\
& \leq \frac{1}{3}\left|f^{\prime}\left(\frac{a+2 b}{3}\right)\right|^{q}+\frac{1}{6}\left|f^{\prime}\left(\frac{a+b}{2}\right)\right|^{q}, \\
& \quad \frac{1}{6}(1-\psi)\left|f^{\prime}\left(\psi b+(1-\psi) \frac{a+2 b}{3}\right)\right|^{q} d \psi \\
& \quad \frac{1}{6}\left|f^{\prime}(b)\right|^{q}+\frac{1}{3}\left|f^{\prime}\left(\frac{a+2 b}{3}\right)\right|^{q} \cdot
\end{aligned}
$$


Combing the above inequalities (2.13), (2.14), (2.15), and (2.16), we obtain (2.12). This completes the proof.

Theorem 2.6 Let $f:[a, b] \subset R \rightarrow R$ be a differentiable function on $I^{0}$, where $a, b \in I$ is with $a<b$. such that $f^{\prime} \in L_{1}([a, b])$. If the mapping $\left|f^{\prime}\right|^{q}$ is concave on $[a, b]$, for some fixed $\mathrm{q}>1$, then we have the following inequality:

$$
\begin{aligned}
& \left|\frac{f\left(\frac{2 a+b}{3}\right)+f\left(\frac{a+2 b}{3}\right)}{2}-\frac{1}{b-a} \int_{a}^{b} f(x) d x\right| \\
& \leq \frac{b-a}{18}\left(\frac{q-1}{2 q-1}\right)^{\frac{q-1}{q}} \mid \\
& \times\left[\left|f^{\prime}\left(\frac{10 a+2 b}{12}\right)\right|+\left|f^{\prime}\left(\frac{7 a+5 b}{12}\right)\right|\right. \\
& \left.+\left|f^{\prime}\left(\frac{5 a+7 b}{12}\right)\right|+\left|f^{\prime}\left(\frac{2 a+10 b}{12}\right)\right|\right] .
\end{aligned}
$$

Proof. Using Lemma 2.1 and well known Hölder inequality we have

$$
\begin{aligned}
& \left|\frac{f\left(\frac{2 a+b}{3}\right)+f\left(\frac{a+2 b}{3}\right)}{2}-\frac{1}{b-a} \int_{a}^{b} f(x) d x\right| \leq \frac{b-a}{9} \\
& {\left[\left(\int_{0}^{1} \psi^{\frac{q}{q-1}} d \psi\right)^{\frac{q-1}{q}}\right.} \\
& \left(\int_{0}^{1}\left|f^{\prime}\left(\psi \frac{2 a+b}{3}+(1-\psi) a\right)\right|^{q} d \psi\right)^{\frac{1}{q}} \\
& +\left(\int_{0}^{1}(1-\psi)^{\frac{q}{q-1}} d \psi\right)^{\frac{q-1}{q}} \\
& \mid\left(\int_{0}^{1}\left|f^{\prime}\left(\psi \frac{a+b}{2}+(1-\psi) \frac{2 a+b}{3}\right)\right|^{q} d \psi\right)^{\frac{1}{q}} \\
& +\left(\int_{0}^{1} \psi^{\frac{q}{q-1}} d \psi\right)^{\frac{q-1}{q}} \\
& \mid\left(\int_{0}^{1}\left|f^{\prime}\left(\psi \frac{a+2 b}{3}+(1-\psi) \frac{a+b}{2}\right)\right|^{q} d \psi\right)^{\frac{1}{q}} \\
& +\left(\int_{0}^{1}(1-\psi)^{\frac{q}{q-1}} d \psi\right)^{\frac{q-1}{q}} \\
& {\left[\left(\int_{0}^{1}\left|f^{\prime}\left(\psi b+(1-\psi) \frac{a+2 b}{3}\right)\right|^{q} d \psi\right)^{\frac{1}{q}}\right]}
\end{aligned}
$$

using the convexity of $\left|f^{\prime}\right|^{q}$, we have

$$
\begin{gathered}
\int_{0}^{1}\left|f^{\prime}\left(\psi \frac{2 a+b}{3}+(1-\psi) a\right)\right|^{q} d \psi \leq\left|f^{\prime}\left(\frac{5 a+b}{6}\right)\right|^{q}, \\
\quad \int_{0}^{1}\left|f^{\prime}\left(\psi \frac{a+b}{2}+(1-\psi) \frac{2 a+b}{3}\right)\right|^{q} d \psi \\
\leq\left|f^{\prime}\left(\frac{7 a+5 b}{12}\right)\right|^{q} \\
\quad \int_{0}^{1}\left|f^{\prime}\left(\psi \frac{a+2 b}{3}+(1-\psi) \frac{a+b}{2}\right)\right|^{q} d \psi \\
\leq\left|f^{\prime}\left(\frac{5 a+7 b}{12}\right)\right|^{q} \\
\int_{0}^{1}\left|f^{\prime}\left(\psi b+(1-\psi) \frac{a+2 b}{3}\right)\right|^{q} d \psi \leq\left|f^{\prime}\left(\frac{a+5 b}{6}\right)\right|^{q} .
\end{gathered}
$$

Combing the above inequalities (2.18), (2.19), (2.20), and (2.21), we obtain (2.17). This completes the proof.

Corollary 2.7 Under the conditions of Theorem 2.6, assume that function is a linear map,

$$
\begin{aligned}
& \left|\frac{f\left(\frac{2 a+b}{3}\right)+f\left(\frac{a+2 b}{3}\right)}{2}-\frac{1}{b-a} \int_{a}^{b} f(x) d x\right| \\
& \leq \frac{b-a}{12}\left(\frac{q-1}{2 q-1}\right)^{\frac{q-1}{q}}\left|f^{\prime}(a+b)\right| .
\end{aligned}
$$

Proof. It is a direct consequence of Theorem 2.6 and using the linearity of the function.

Theorem 2.8 Let $f:[a, b] \subset R \rightarrow R$ be a differentiable function on $I^{0}$, where $a, b \in I$ is with $a<b$. such that $f^{\prime} \in L([a, b])$. If the mapping $\left|f^{\prime}\right|^{q}$ is concave on $[a, b]$, for some fixed $q \geq 1$, then we have the following inequality :

$$
\begin{aligned}
& \left|\frac{f\left(\frac{2 a+b}{3}\right)+f\left(\frac{a+2 b}{3}\right)}{2}-\frac{1}{b-a} \int_{a}^{b} f(x) d x\right| \\
& \left.\leq \frac{b-a}{18}\left[\left|f^{\prime}\left(\frac{14 a+4 b}{18}\right)\right|+\left|f^{\prime}\left(\frac{11 a+7 b}{18}\right)\right|\right]+\left|f^{\prime}\left(\frac{7 a+11 b}{18}\right)\right|+\left|f^{\prime}\left(\frac{4 a+14 b}{18}\right)\right|\right] .
\end{aligned}
$$

Proof. By the concavity of $\left|f^{\prime}\right|^{q}$ and Power-mean inequality we have

$$
\begin{aligned}
& \left|f^{\prime}(\psi x+(1-\psi) y)\right|^{q} \geq \psi\left|f^{\prime}(x)\right|^{q}+(1-\psi)\left|f^{\prime}(y)\right|^{q} \\
& \geq\left(\psi\left|f^{\prime}(x)\right|+(1-\psi)\left|f^{\prime}(y)\right|\right)^{q}
\end{aligned}
$$


And thus

$$
\left|f^{\prime}(\psi x+(1-\psi) y)\right| \geq \psi\left|f^{\prime}(x)\right|+(1-\psi)\left|f^{\prime}(y)\right|,
$$

Using Lemma 2.1 and the Jensen's integral inequality, we have

$$
\left.\begin{array}{c}
\frac{f\left(\frac{2 a+b}{3}\right)+f\left(\frac{a+2 b}{3}\right)}{+\int_{0}^{1}(\psi-1)}-\frac{1}{b-a} \int_{a}^{b} f(x) d x \leq \frac{b-a}{9} \times \\
+\int_{0}^{1} \psi f^{\prime}\left(\psi f^{\prime}\left(\psi \frac{2 a+b}{2}+(1-\psi) \frac{2 a+b}{3}\right) d \psi\right. \\
\left.+\frac{a+2 b}{3}+(1-\psi) a\right) d \psi
\end{array}\right]
$$

Corollary 2.9 Under the conditions of Theorem 2.8,

$$
\left|\frac{f\left(\frac{2 a+b}{3}\right)+f\left(\frac{a+2 b}{3}\right)}{2}-\frac{1}{b-a} \int_{a}^{b} f(x) d x\right| \leq \frac{b-a}{18}\left|f^{\prime}(a+b)\right|
$$

Proof. The assertion is a direct consequence of Theorem 2.8 and using the linearity of function.

\section{Application to Some Special Means}

Let us recall the following means for arbitrary real numbers $a$ and $b$.

The Arithmetic mean

$$
A=A(a, b)=\frac{a+b}{2}, a, b \geq 0 .
$$

The Harmonic mean

$$
A=A(a, b)=\frac{2 a b}{a+b}, a, b \geq 0 .
$$

Generalized-logarithmic mean

$$
L_{n}(a, b)=\left\{\begin{array}{ll}
a, & \text { if } a=b \\
{\left[\frac{b^{n+1}-a^{n+1}}{(n+1)(b-a)}\right]^{\frac{1}{n}},} & \text { if } a \neq b
\end{array} .\right.
$$

The Logarithmic mean

$$
L=L(a, b)=\left\{\begin{array}{ll}
a, & \text { if } a=b \\
\frac{b-a}{\ln b-\ln a}, & \text { if } a \neq b
\end{array} .\right.
$$

Now utilizing outcomes of Section 2, some new inequalities are derived for the above means.

Proposition 3.3.1. Let $a, b \in R^{+}, a<b$, and $n \in R$. then, we have

$$
\begin{aligned}
& \left|A\left(\left(\frac{2 a+b}{3}\right)^{n},\left(\frac{a+2 b}{3}\right)^{n}\right)-L_{n}^{n}(a, b)\right| \\
& \leq|n|\left(\frac{b-a}{9}\right) A\left\{|a|^{n-1},|b|^{n-1}\right\} .
\end{aligned}
$$

Proof. By corollory 2.3 applied for the mapping $f(x)=x^{n}, \quad x \in R$, we have the above inequality (3.1).This completes the proof.

Proposition 3.3.2. Let $a, b \in R^{+}, a<b$, and $n \in R$. then, we have

$$
\left|H^{-1}\left(\frac{2 a+b}{3}, \frac{a+2 b}{3}\right)-L(a, b)\right| \leq \frac{b-a}{9} A\left(|a|^{-2},|b|^{-2}\right)
$$

Proof. By corollory 2.3 applied for the mapping $f(x)=\frac{1}{x}, x \in R$, we have the above inequality (3.2). This completes the proof.

\section{Application to Quadrature Formula}

Let $D$ be the partition $\left\{a=x_{0}<x_{1}<\ldots .<x_{n-1}<x_{n}=b\right\}$ of the interval $[a, b]$, and consider the quadrature formula

$$
\int_{a}^{b} f(x) d x=Q(f, D)+E(f, D)
$$


where

$$
Q(f, D)=\frac{1}{2} \sum_{k=0}^{n-1}\left[\begin{array}{c}
f\left(\frac{2 x_{i}+x_{i+1}}{3}\right) \\
+f\left(\frac{x_{i}+2 x_{i+1}}{3}\right)
\end{array}\right]\left(x_{i+1}-x_{i}\right) .
$$

For the quadrature version and $E(f, D)$ denotes the approximation error.

Proposition 4.1. Let $f:[a, b] \subset R \rightarrow R$ be a differentiable mapping on $I^{0}$ ( interior of $I^{0}$ ) $a, b \in I$ with $a<b$. If the mapping $\left|f^{\prime}\right|$ is convex on $[a, b]$, then for every division $D$ of $[a, b]$, the following holds:

$$
\left.\begin{array}{l}
|E(f, D)| \leq \frac{1}{54}\left(x_{i+1}-x_{i}\right)^{2} \\
\times \sum_{k=0}^{n-1}\left[\left|f^{\prime}\left(x_{i}\right)\right|+4\left|f^{\prime}\left(\frac{2 x_{i}+x_{i+1}}{3}\right)\right|+2\left|f^{\prime}\left(\frac{x_{i}+x_{i+1}}{2}\right)\right|\right] \\
+4\left|f\left(\frac{x_{i}+2 x_{i+1}}{3}\right)\right|+\left|f^{\prime}\left(x_{i+1}\right)\right|
\end{array}\right]
$$

Proof. Using Theorem 2.2 on the subintervals $\left[x_{i}, x_{i+1}\right]$ $(i=0,1, \ldots, n-1)$ of the division $D$, we have

$$
\left.\begin{array}{l}
\left|\frac{1}{2}\left[f^{\prime}\left(\frac{2 x_{i}+x_{i+1}}{3}\right)+f^{\prime}\left(\frac{x_{i}+2 x_{i+1}}{3}\right)\right]\right| \leq\left(\frac{x_{i+1}-x_{i}}{54}\right) \\
-\frac{1}{x_{i+1}-x_{i}} \int_{x_{i}}^{x_{i+1}} f(x) d x \\
{\left[\left|f^{\prime}\left(x_{i}\right)\right|+4\left|f^{\prime}\left(\frac{2 x_{i}+x_{i+1}}{3}\right)\right|+2\left|f^{\prime}\left(\frac{x_{i}+x_{i+1}}{2}\right)\right|\right] .} \\
+4\left|f\left(\frac{x_{i}+2 x_{i+1}}{3}\right)\right|+\left|f^{\prime}\left(x_{i+1}\right)\right|
\end{array}\right] .
$$

$$
\begin{aligned}
& |E(f, D)|= \\
& \left|\sum_{i=0}^{n-1} \int_{x_{i}}^{x_{i+1}} f(x) d x-\frac{1}{2} \sum_{i=0}^{n-1}\left[\begin{array}{l}
f\left(\frac{2 x_{i}+x_{i+1}}{3}\right) \\
+f\left(\frac{x_{i}+2 x_{i+1}}{3}\right)
\end{array}\right]\left(x_{i+1}-x_{i}\right)\right| \\
& \leq \sum_{i=0}^{n-1}\left(x_{i+1}-x_{i}\right)\left|\begin{array}{l}
\frac{1}{2}\left[f\left(\frac{2 x_{i}+x_{i+1}}{3}\right)+f\left(\frac{x_{i}+2 x_{i+1}}{3}\right)\right] \mid \\
-\frac{1}{x_{i+1}-x_{i}} \int_{x_{i}}^{x_{i+1}} f(x) d x
\end{array}\right|
\end{aligned}
$$

By combining (4.2) and (4.3), we obtain (4.1). This completes the proof.

\section{References}

[1] C. Niculescu, L. E. Persson, Convex functions and their application, Springer, Berlin Heidelberg New York, (2004).

[2] M. W. Alomari, M. Darus, U.S. Kirmaci. Some inequalities of Hermite-Hadamard type for s-convex functions. Acta Math. Sci. Ser. B Engl. Ed. 31 (4), 1643-1652 (2011).

[3] S. S. Dragomir, R. P. Agarwal. Two inequalities for differentiable mappings and applications to special means of real numbers and to trapezoidal formula, Appl. Math. Lett., 11 (5) (1998) 91-95.

[4] U. S. Kirmaci, M. E. Özdemir. On some inequalities for differentiable mappings and applications to special means of real numbers and to midpoint formula, Appl. Math. Comput., 153 (2004) 361-368.

[5] U.S. Kirmaci. Inequalities for differentiable mappings and applications to special means of real numbers and to midpoint formula, Appl. Math. Comp., 147 (1) (2004), 137-146.

[6] H. Kavurmaci, M. Avci, M. E. Özdemir. New inequalities of Hermite-Hadamard type for convex functions with applications ar Xiv: 1006. $1593 \mathrm{vl}$ [math. CA]

[7] H. Hudzik, L. Maligrada. Some remarks on s-convex functions, Aequationes Math. 48 (1994) 100-111.

[8] S. S. Dragomir, C. E. M. Pearce. Selected Topic on HermiteHadamard Inequalities and Applications, Melbourne and Adelaide, December, 2000.

[9] S. S. Dragomir, S. Fitzpatrick. The Hadamard's inequality for sconvex functions in the second sense, Demonstratio Math. 32 (4) (1999), 687-696.

[10] U. S. Kirmaci, K. Klaričić Bakula, M. E. Özdemir, J. Pečarić. Hadamard-type inequalities for s-convex functions, Appl. Math. Comput., 193 (1) (2007) 26-35.

[11] M. Tunç. On some new inequalities for convex functions, Turk $\mathrm{J}$ Math 35 (2011), 1-7.

[12] M. Tunç. New integral inequalities for s-convex functions, RGMIA Research Report Collection Volume 13, Issue 2, 2010.

[13] R.-F. Bai, F.Qi, B.-Y. Xi. Hermite-Hadamard type inequalities for the $\mathrm{m}$-and $(\alpha, \mathrm{m})$-logarithmically convex functions. Filomat $27(1)$, 1-7 (2013).

[14] S.-P. Bai, F. Qi. Some inequalities for (s 1, m 1)-(s 2, m 2)convex functions on the co-ordinates. Glob. J. Math. Anal. 1 (1), 22-28 (2013).

[15] B.-Y. Xi, F. Qi. Hermite-Hadamard type inequalities for functions whose derivatives are of convexities. Nonlinear Funct. Anal. Appl. 18 (2), 163-176 (2013).

[16] S. Qaisar, C. He. S. Hussain. On New Inequalities of HermiteHadamard type for generalized Convex Functions. Italian journal of pure and applied mathematics. In Press. 